BMC

Bioinformatics

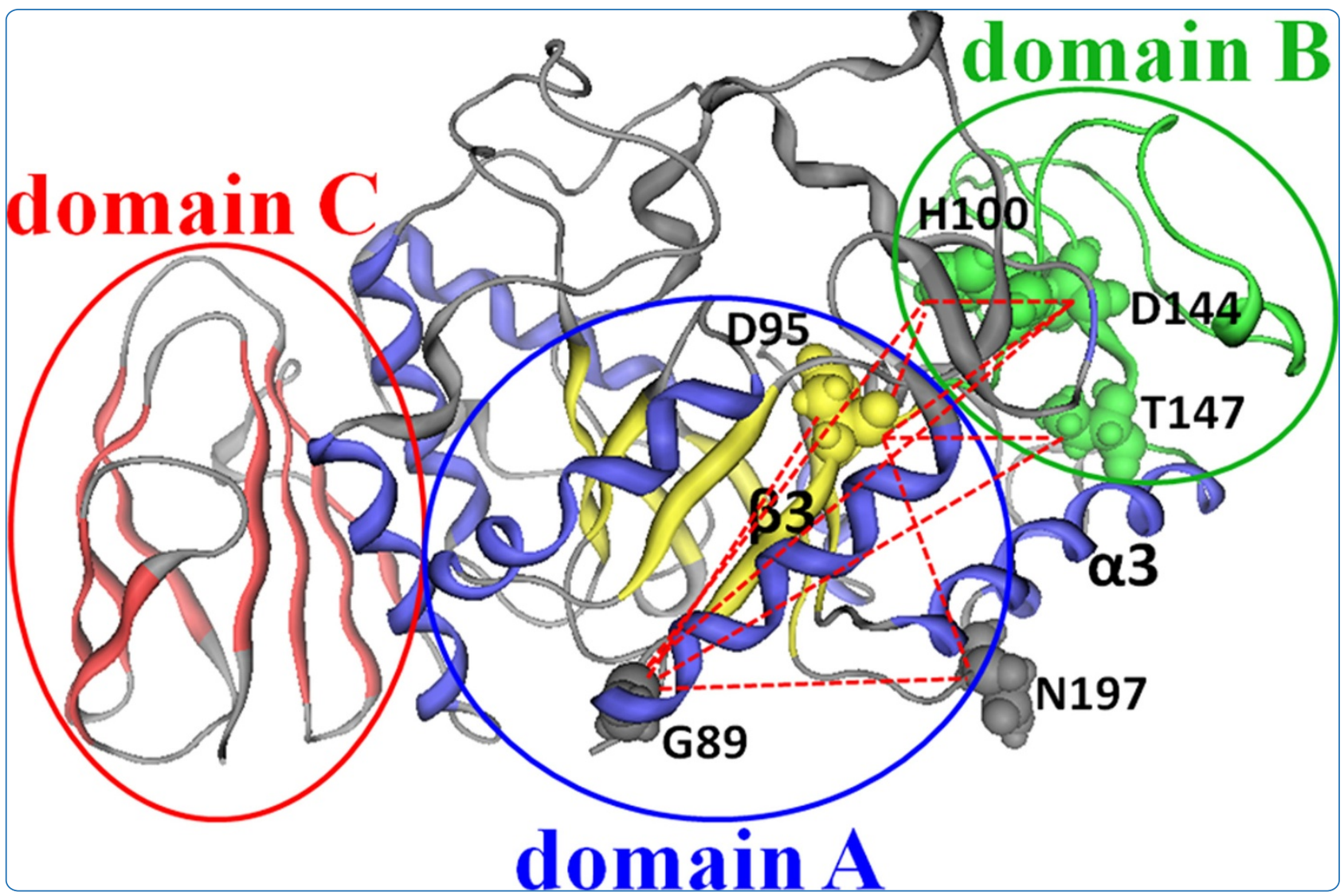

Improving the thermostability of alpha-amylase by combinatorial coevolving-site saturation mutagenesis

Wang et al.

C Biomed Central

Wang et al. BMC Bioinformatics 2012, 13:263

http://www.biomedcentral.com/1471-2105/13/263 


\title{
Improving the thermostability of alpha-amylase by combinatorial coevolving-site saturation mutagenesis
}

\author{
Chenghua Wang ${ }^{1,2}$, Ribo Huang ${ }^{1,2,3^{*}}$, Bingfang $\mathrm{He}^{1}$ and Qishi Du ${ }^{2,3}$
}

\begin{abstract}
Background: The generation of focused mutant libraries at hotspot residues is an important strategy in directed protein evolution. Existing methods, such as combinatorial active site testing and residual coupling analysis, depend primarily on the evolutionary conserved information to find the hotspot residues. Hardly any attention has been paid to another important functional and structural determinants, the functionally correlated variation informationcoevolution.
\end{abstract}

Results: In this paper, we suggest a new method, named combinatorial coevolving-site saturation mutagenesis (CCSM), in which the functionally correlated variation sites of proteins are chosen as the hotspot sites to construct focused mutant libraries. The CCSM approach was used to improve the thermal stability of a-amylase from Bacillus subtilis CN7 (Amy7C). The results indicate that the CCSM can identify novel beneficial mutation sites, and enhance the thermal stability of wild-type Amy $7 \mathrm{C}$ by $8^{\circ} \mathrm{C}\left(T_{50}^{30}\right)$, which could not be achieved with the ordinarily rational introduction of single or a double point mutation.

Conclusions: Our method is able to produce more thermostable mutant a-amylases with novel beneficial mutations at new sites. It is also verified that the coevolving sites can be used as the hotspots to construct focused mutant libraries in protein engineering. This study throws new light on the active researches of the molecular coevolution.

\section{Background}

Directed protein evolution is invaluable in engineering biocatalysts for better properties, such as enhancements in activity, stability, and enzyme selectivity $[1,2]$. However, it is usually limited by its inability to generate highquality mutant libraries containing more beneficial variants. This is especially problematic considering the combinatorial complexity of mutant libraries and the huge sequence space $[3,4]$. Constructing focused mutant libraries at defined hotspot residues is considered to be one of the most promising ways of improving directed protein evolution [3-5]. Much of pioneering work has been complemented by Reetz's team [6-8].

\footnotetext{
* Correspondence: rbhuang@gxas.ac.cn

'Biotechnology and Pharmaceutical Engineering, Nanjing University of Technology, Xinmofan Road 5, Nanjing 210009, Jiangsu, China

${ }^{2}$ State Key Laboratory of Enzyme Technology, National Engineering Research Center for Non-food Biorefinery, Guangxi Key Laboratory of Biorefinery, Guangxi Academy of Sciences, Nanning, Guangxi 530007, China Full list of author information is available at the end of the article
}

All existing focused mutant library methods can be essentially classified into two categories: structure-based approaches and sequence-based approaches. The former includes combinatorial active site testing (CAST), B-factors, and knowledge-based potential analysis [6-10]. The latter includes protein design automation (PDA) [11], residual coupling analysis (RCA) [12], and ConSurf [13]. While the aforementioned methods depend primarily on the evolutionary conservation information to find out the hotspot residues, there are some other important functional and structural determinants desirable to be taken into consideration, such as the functionally correlated variation information-coevolution.

Co-evolution is the correlated variation of protein sites promoted by selective pressures [14]. The cooperation between residues at the coevolving sites, which usually takes the form of compensatory interactions, synergistic effects, allosteric interactions, and epistatic interactions [15-19], determines the structure and function of

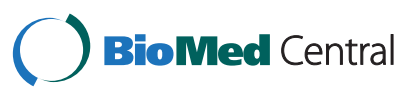


proteins [20,21]. In recent years much attention has been paid to find the coevolving residues, as well as the reasons why residues co-evolve [14,21-28], but few experimental design methods based on the coevolution and successful examples of using them have been reported.

In this study, we propose a method, combinatorial coevolving-site saturation mutagenesis (CCSM), which chooses the coevolving sites of proteins as hotspot residues to construct focused mutant libraries. We also describe the successful use of the CCSM method to improve the thermostability of $\alpha$-amylase.

\section{Results and discussion}

$\alpha$-Amylase is an important industrial biocatalyst in starch liquefaction processes and a valuable model enzyme for studies of thermal adaptation in proteins [29]. We used the CCSM approach to improve the thermostability of $\alpha$-amylase (Amy7C) to demonstrate the feasibility of this method.

\section{Spotting the coevolving sites in Amy7C}

Six coevolving residues and 10 pairs of co-evolutionary interactions were identified in Amy7C during step 1 of the CCSM approach (see Additional file 1, Additional file 2: Table SA2 and Additional file 3: Table SA3 for computational details). As shown in Figure 1, among the six residues, H100, D144, and T147 are located in domain B, and G89, D95 and N197 are in domain A. In domain A, the G89 is at the loop linking $\alpha 2$ and $\beta 3$, D95 is on $\beta 3$, and N197 is at the loop linking $\alpha 4$ and $\beta 5$. Except for D95, all the coevolving sites are situated exactly

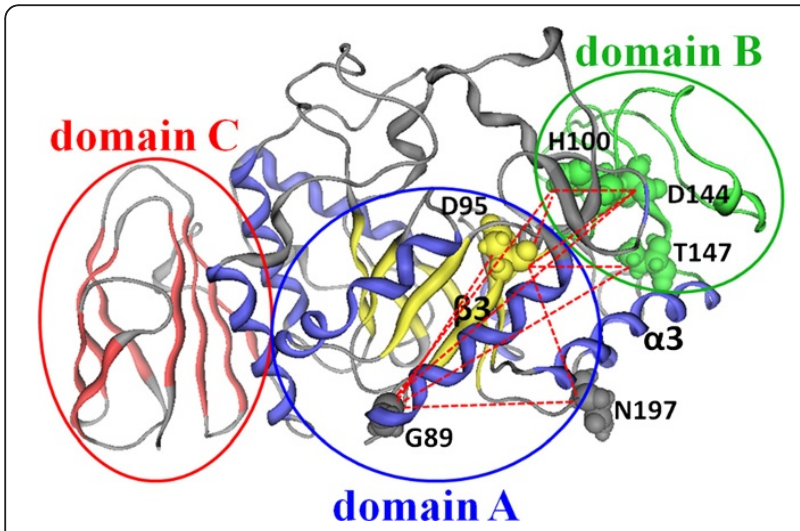

Figure 1 Distribution of co-evolving sites in Amy7C. Amy $7 C$ is shown in cartoon form, and the coevolving sites are shown in filled balls. The catalytic domain A, consisting of a closed eight-stranded parallel $\beta$ sheet barrel (yellow) surrounded by eight a helices (blue), is circled in blue. Domain $B$ that protrudes between third $\beta$-strand and third a-helix of domain A, is circled in green. The C-terminal $\beta$ sheet (red), domain $C$ is circled in red. The sequences linking the domains are shown in gray. The dashed red line indicates the coevolutionary relationship between each pair of co-evolving sites. in the so-called "stability face" of Amy7C. This stability face includes domain $\mathrm{B}$ and the loops linking the $\alpha$ helices with the subsequent $\beta$ strands of TIM barrel of domain A [30]. The above observation of coevolving sites is consistent with previously published works, which demonstrated the thermostable mutations concentrated on the stability face, by conventional blind or rational protein engineering experiments [31]. However, the coevolving sites of Amy7C spotted by us in this work are distributed across a larger region than the stability face defined by other authors [30], and they are different sites from those identified by other authors [31].

The average distance between all coevolving sites in Amy7C is in the range $17.3 \pm 7.31 \AA$, which is much greater than that reported by other research teams $[26,32]$. The distance between coevolving sites are significantly greater than the distance used to define hotspot sites in previous studies, which is usually about $5 \AA$ $[6,11]$. The differences between the coevolving sites in this study and the hotspot sites found by previous studies must be attributed to the prediction methods, because the previous studies identified hotspots by evolutionary conservative information-based methods, such as the sequence alignment-based method and distance-based method [6-8], which could not usually find the coevolving sites located as distant as $>17 \AA$ apart.

\section{Construction and screening of CCSM libraries}

Ten CCSM libraries were constructed at coevolving-sites and explored using the HTS method, which is based on the starch-iodine method and DNS method [33,34] (see Additional file 1 for details). All possible combinations and permutations of amino acid residues are explored in the CCSM library through simultaneous and random mutation of the coevolving-sites using the $\mathrm{NNK}(\mathrm{G} / \mathrm{T})$ degenerate primers (see Table SA1 in Additional file 4: Table SA1).

A total of 10,010 clones were randomly selected and screened using the starch-iodine method in the first screening. The majority of the variants displayed impaired activities, and only about $10 \%$ retained any obvious starch hydrolytic ability relative to parental Amy7C. The active variants made up less than 5\% of the three libraries of G89H100, G89D144, and G89T147. Active variants of the other seven libraries made up around $12.5 \%$.

A total of 880 potential hits in the initial screening were rescreened by the DNS method using freshly transformed cells to discard false positives. In the 880 variants, 152 variants showed above $10 \%$ of the parent enzyme's activity, and only 76 variants displayed more than $50 \%$ activities. The activity landscape of the top 152 variants is shown in Figure 2. It can be seen that the top 


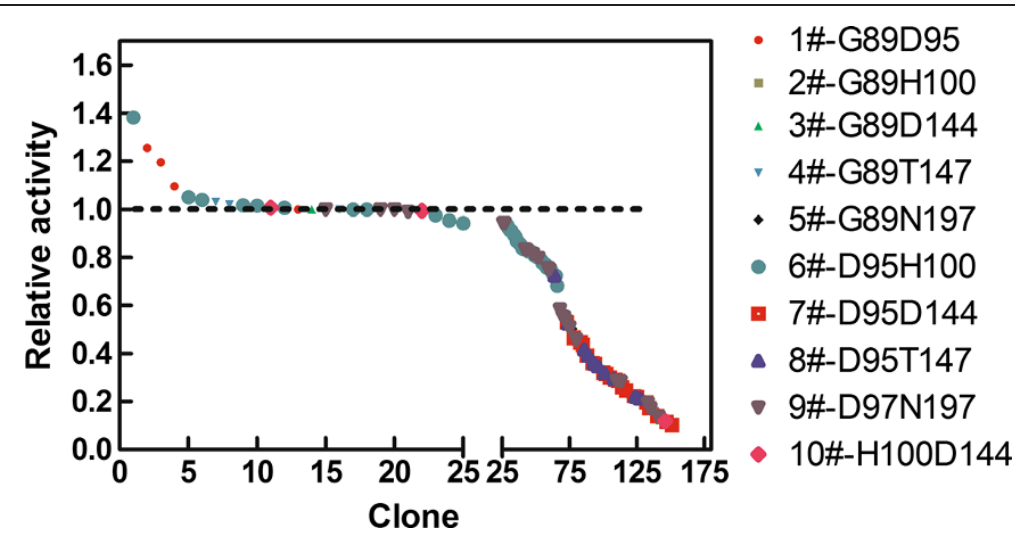

Figure 2 Activity landscape of the CCSM libraries plotted in descending order. The dotted line indicates relative activity of the wild type amylase, which is scaled to 1 . The top 25 variants are shown in the first segment of the horizontal axis, the other 127 variants are shown in the second segment.

25 variants, as shown around the dotted line in the first segment of the horizontal axis in Figure 2, mainly came from D95H100, G89D95 and D97N197 libraries, while variants from the H100D144, D95T147 and D95D144 libraries showed relatively low activities.

\section{Rescreening of CCSM libraries}

The top 120 variants (12 variants in each CCSM library) were rescreened by characterizing their relative activities and their $T_{50}^{30}$ values compared to the wild type, using freshly prepared crude enzymes. The average relative activity of the 120 variants was found to be $0.68 \pm 0.28$, in contrast to the $1.02 \pm 0.22$ of wild-type enzyme. The average $T_{50}^{30}$ value of the 120 variants was found to be $63.5 \pm 2.86^{\circ} \mathrm{C}$, compared to $64.8 \pm 1.04^{\circ} \mathrm{C}$ of the wild-type enzyme. A total of 98 variants had half-inactivating temperature above $58^{\circ} \mathrm{C}$ and retained more than $10 \%$ relative activity in comparison to the wild-type enzyme.
Figure 3 depicts the relative activity and $T_{50}^{30}$ values of the top 98 in the 120 variants. From the Figure 3, we can see that among the 24 most thermostable variants compared with the wild type, 16 contained one of the H100, D144 and T147 sites, so it appears that these three sites in domain $\mathrm{B}$ are primarily responsible for the most thermally stable variants.

\section{Sequence analysis of the CCSM mutants}

The sequence analysis of top 98 variants in the rescreening indicated that 28 variants $(28.6 \%)$ had not changed at all (could be regarded as false positive), 35 variants (35.7\%) had mutated at single sites, and 35 variants (35.7\%) had double mutations at the designed coevolving sites. Table 1 summarizes the amino acids and codons distributed over each site. Most of the mutations observed in our CCSM library require a minimum of two nucleotide changes per codon, and some can only

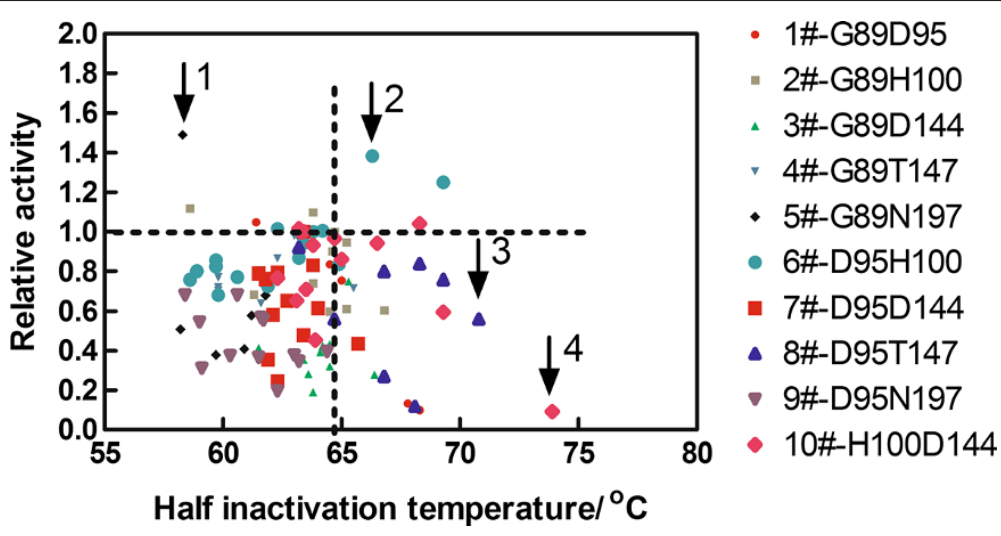

Figure 3 The relative activity and $\mathrm{T}_{50}^{30}$ value distribution of variants in the CCSM libraries. Relative activities (vertical axis) of variants are plotted versus $T_{50}^{30}$ values (horizontal axis). The four representative variants N197C, H100I, T147P, and H100MD144R are marked out by "1", "2", "3", and " 4 ", respectively. The horizontal dotted line represents the relative activity of the wild type amylase, and the vertical one denotes its half inactivation temperature $\left(T_{50}^{30}\right)$. 
Table 1 The distribution of amino acids and codons at the 6 coevolving sites in the sequenced 92 amylase variants

\begin{tabular}{|c|c|c|c|c|c|c|c|}
\hline \multirow{2}{*}{$\begin{array}{l}\text { Amino } \\
\text { Acid }\end{array}$} & \multicolumn{6}{|c|}{ Positions } & \multirow[b]{2}{*}{ No. } \\
\hline & G89 & D95 & $\mathrm{H} 100$ & D144 & T147 & N197 & \\
\hline W. T. & GGC & GAT & CAT & GAT & $\mathrm{ACT}$ & AAT & \\
\hline Ala (A) & $\underline{\mathrm{GCG}}_{2}$ & & & & & $\mathrm{CGC}_{1}$ & 3 \\
\hline Cys (C) & $\underline{\mathrm{TTT}}_{2}$ & & & & $\underline{\mathbf{T G T}}_{3}$ & $\underline{\mathrm{TGT}}_{7}$ & 12 \\
\hline $\begin{array}{l}\text { Asp } \\
\text { (D) }\end{array}$ & $\underline{\mathbf{C T G}}_{1}$ & & & & & $\underline{\mathbf{G}} \mathrm{AT}_{1}$ & 2 \\
\hline Glu (E) & 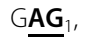 & & & $\mathrm{GA} \underline{\mathbf{G}}_{3}$ & & & 4 \\
\hline Phe (F) & $\underline{\mathbf{T T T}}_{1}$ & & & & & & 1 \\
\hline Gly (G) & $\begin{array}{l}\mathrm{GG} \mathbf{G}_{5 \prime} \\
\mathrm{GG}^{\prime} \underline{\mathbf{T}}_{3}\end{array}$ & & & $\frac{\mathbf{G G G}_{4},}{\mathrm{GG}_{4}}$ & & $\underline{\mathrm{GGG}}_{3}$ & 27 \\
\hline $\mathrm{His}(\mathrm{H})$ & & $\underline{\mathbf{C}} \mathrm{AT}_{1}$ & & & & & 1 \\
\hline Ile (I) & & & $\underline{\mathbf{A T T}}_{2}$ & & & & 2 \\
\hline Lys (K) & & & & & & $\mathrm{AA} \underline{\mathbf{G}}_{1}$ & 1 \\
\hline Leu (L) & $\underline{\mathrm{CTG}}_{1}$ & & & & & & 1 \\
\hline $\begin{array}{l}\text { Met } \\
\text { (M) }\end{array}$ & & & $\underline{\mathrm{ATG}}_{4}$ & & & $\underline{A T G}_{2}$ & 6 \\
\hline $\begin{array}{l}\text { Asn } \\
\text { (N) }\end{array}$ & $\underline{\mathbf{A A T}}{ }_{1}$ & & $\underline{\mathbf{A}} \mathrm{A} \mathrm{T}_{2}$ & & $\mathrm{~A} \underline{\mathbf{A}}_{3}$ & & 6 \\
\hline Pro (P) & & & & & $\underline{\mathbf{c}} \mathrm{CT}_{3}$ & & 3 \\
\hline Gln (Q) & $\underline{\text { CAG }}_{1}$ & & $\mathrm{CA} \underline{\mathbf{G}}_{1}$ & & & $\underline{\mathbf{C}} \underline{A}_{1}$ & 3 \\
\hline Arg (R) & $\begin{array}{l}\mathbf{\mathbf { C }} G \mathbf{T}_{31} \\
\overline{\mathbf{C}} G \overline{\mathrm{C}}_{3}\end{array}$ & $\underline{\mathbf{C G G}}_{1}$ & $\mathrm{CGG}_{1}$ & $\mathbf{C G G}_{1}$ & $\begin{array}{l}\mathrm{CGT}_{4,} \\
\overline{\mathrm{CGC}}_{3}\end{array}$ & $\mathrm{AGG}_{1}$ & 20 \\
\hline Ser $(S)$ & & & $\underline{\mathbf{A G T}}_{1}$ & & $\underline{\mathbf{T}} \mathrm{CT}_{1}$ & $\frac{\mathbf{T C G}_{5}}{\mathrm{AG}_{1}}$ & 3 \\
\hline Thr $(T)$ & $\underline{\mathbf{A C A}}_{1}$ & & & & & & 1 \\
\hline Val (V) & & & & $\mathrm{GIT}_{3}$ & $\underline{\mathbf{G T G}}_{3}$ & & 6 \\
\hline $\begin{array}{l}\text { Trp } \\
(\mathrm{W})\end{array}$ & & & & $\underline{\mathbf{T G G}}_{1}$ & & & 1 \\
\hline Tyr (Y) & & & & & & & 0 \\
\hline Type & 11 & 2 & 6 & 5 & 6 & 9 & \\
\hline
\end{tabular}

Subscript numbers represent the frequencies of the codons in the 92 sequenced variants. Underlined bold letters indicate substitution to the wild type nucleotide.

No. $=$ total number of codons different from the wild type for each amino acid. Type = type number of amino acids appeared at each site. W. T. = wild type.

be by more than three nucleotide changes (Table 1). These nucleotide changes are nearly inaccessible for conventional error-prone PCR and single-gene DNA shuffling methods [11].

All the coevolving sites showed dramatic variation in either single or double mutations, except D95 showed only two double mutations, i.e., D95HT147S (CATTCT) and G89FD95R (TTTCGG). G89 and N197 were found to be the most diverse mutation sites, which displayed 11 and 9 different kinds of amino acids respectively (Table 1). Previous studies have shown that the eight strands and eight helixes of the TIM barrel of domain A are vital to the stability of the structure [35,36], and few beneficial mutations can exist there. In this study, both D95HT147S and G89FD95R were found to involve changes to the residue D95 of the $\beta 3$ in the TIM barrel of the domain A. The detrimental effects caused by D95 site mutation must be compensated by the covariation at the other coevolving site, like the T147S in D95HT147S. The similar but beneficial cooperation may also take place between coevolving residues in improved variants. The positions and interactions between coevolving residues in some example variants are shown in Figure A1 (see Additional file 5: Figure SA1).

The aforementioned "false positive" phenomenon of high percentages of same sense mutations (28.6\%) and single mutants (35.7\%) upon rescreening should probably be attributed to the relatively lenient criteria adopted in our library construction and screening procedures. NNK degeneracy in the primers offers a variety of 32 codons and encodes all possible 20 amino acids, so it will inevitably produce same sense mutations in the library construction. Meanwhile, the selection criteria for the sequenced 98 variants were set at above $58^{\circ} \mathrm{C}$ and at more than $10 \%$ residual relative activity, which are far below that (about $64.8^{\circ} \mathrm{C}$ and $50 \%$ ) of the wild-type enzyme (Figure 3).

\section{Validation of the representative improved variants}

To evaluate the effects of CCSM in improving the thermal stability of Amy7C, the wild-type Amy7C and four representative variants of N197C, H100I, T147P and H100MD144R (denoted by "1", "2", "3" and "4" in Figure 3), were purified to homogeneity and characterized [see Addition file 1]. There appeared to be a tradeoff between thermal stability and catalytic activity of Amy7C variants[37]. Amy7C showed a $k_{\text {cat }}$ value of $1260.55 \mathrm{~s}^{-1}$ and a $T_{50}^{30}$ value of $62.3^{\circ} \mathrm{C}$. N197C showed a reduced $T_{50}^{30}$ value of $58.3^{\circ} \mathrm{C}$ and a slightly higher catalytic activity $k_{\text {cat }}$ value of $1298.37 \mathrm{~s}^{-1}$. From the H100I, to T147P, to H100MD144R, the $T_{50}^{30}$ values increased by $4.5^{\circ} \mathrm{C}, 7^{\circ} \mathrm{C}$, and $8^{\circ} \mathrm{C}$, while the catalytic activities range from 1.04-fold, to 0.74 -fold, to 0.31 fold, respectively.

Due to both the academic and industrial values, amylase has been extensively studied in different laboratories, and numerous engineering work has been done to increase the thermostabiliy [38]. Among the most excellent works, Machius et al. have successfully identified some beneficial amino acid substitutions in an amylase BLA from Bacillus licheniformis [39-44], and even created a hyperthermostable variant with $23^{\circ} \mathrm{C}$ higher than the wild-type enzyme by substituting 7 amino acids $[31,38,44]$. However, to the best of our knowledge, if the test conditions and sources of $\alpha$-amylases are not considered, the $T_{50}^{30}$ increase of $8^{\circ} \mathrm{C}$ 
observed in this study is the largest ever achieved with a single round by introducing up to two point mutations into wild-type $\alpha$-amylases [31].

As a coevolving strategy, our method also identified stabilizing variants with only single mutations at certain coevolving sites, such as H100I and T147P mutations (see above). From time to time, there is no difference between our coevolving method and traditional mutation methods such as error-prone PCR and DNA shuffling in generation of stabilizing single mutations, but in fact our single mutation should be regarded as same as other coevolving double mutations since the newly introduced single amino acid has somehow improved the coordination between two residues at the coevolving sites, and made them perfectly match in certain performances such as thermostability.

So, the above validation results clearly indicate that the screened beneficial variants changed at the coevolving sites, and the new amino acid combinations and the cooperation between them at coevolving sites brought greater thermal stability than the wild-type enzyme. It also indicates that CSSM may be more effective in generating desired mutations because it involves at least two coevolving sites that may be located in some far-away positions in protein sequences but more likely in the proximity to each other on the three dimensional structure of the proteins, and since it involves the coordinate changes in both amino acid positions they will then be more likely to coevolve towards some direction we desired, which could be imagined as coordinated "directed evolution", in sharp contrast to the ordinary "directed evolution". The method proposed here only uses the protein sequence to detect coevolving sites, then employs combinatorial saturation mutagenesis to create mutations changing at both coevolving sites, and then screens out beneficial variants. So, it seems promising that the CSSM method should be applicable to many interesting enzymes other than $\alpha$-amylase.

\section{Conclusions}

This study shows that the new method of choosing the coevolving sites as the hotspots for constructing focused mutant libraries leads to improved variants with novel beneficial mutations at new sites. The successful application of CCSM in improving the thermostability of $\alpha$ amylase in this study also throws new light on the active researches of the molecular coevolution.

\section{Methods}

The CCSM approach combines coevolving site identification with combinatorial saturation mutagenesis [45] and high throughput screening method. The CCSM approach is carried out in three steps.

\section{Step 1: Identification of coevolving sites}

The coevolving sites in protein families and the coevolving pairs of residues in a query protein sequence are predicted by carrying out the following five methods successively, according to the state-of-the-art row and column weighting of mutual information (RCW-MI) method [22]. Firstly, the query sequence is compared to the Uniprot [46] by BLASTP, and the compatible homologous sequences are retrieved. Secondly, the homologous sequences are aligned via the MAFFT [47] method to build the family sequence alignment. Thirdly, the alignment is then processed by MaxAlign [48] to diminish the number of gapped columns in the alignment. Fourthly, the mutual information between each two sites is calculated by the equation (1) [22], which consists of the mutual information matrix.

$$
M I(A: B)=\sum_{i} \sum_{j} P\left(a_{i}, b_{j}\right) \log _{20}\left[\frac{P\left(a_{i}, b_{j}\right)}{P\left(a_{i}\right) P\left(b_{j}\right)}\right]
$$

Where, $\operatorname{MI}(A: B)$ is the mutual information between two sites $A$ and $B$, and $\mathrm{i}$ and $\mathrm{j}$ run through all the occurring amino acids in each site. The base 20 for the logarithm is the number of letters in the protein alphabet. $P$ $\left(a_{i}\right), P\left(b_{j}\right)$ and $P\left(a_{i}, b_{j}\right)$ are the observed frequencies of amino acids $a_{i}, b_{j}$ and $\left(a_{i}, b_{j}\right)$, respectively.

Fifthly, each site pair of the mutual information matrix is weighted by the average score of constituting sites according to according to the equation (2) [49].

$$
R C W(A: B)=\frac{M I_{i j}}{\left(M I_{i .}+M I_{. j}-2 M I_{i j}\right) /(n-1)}
$$

Where, $R C W(A: B)$ is the row and column weighted mutual information between $A$ and $B$ sites, $M I_{i j}$ represents the mutual information between sites $i$ and $j, M I_{i}$. stands for the summation over all sites in row $i, M I_{. j}$ denotes the sum of the Mutual Information matrix over all lines in column $j, n$ is the number of alignment sequences.

The coevolving sites prediction in this research was carried out by the above method via InterMap3D server [50], which is an available server to the general community for predicting and visualizing co-evolving proteins residues.

\section{Step 2: Construction of combinatorial saturation mutagenesis library at coevolving sites}

The CCSM libraries are constructed by simultaneously and randomly mutating the coevolving sites using the protocol of QuickChange ${ }^{\odot}$ XL Site-Directed Mutagenesis Kit from Stratagene (La Jolla, CA) [51]. Complementary primers 33-35 nucleotides in length, which include NNK (G/T) degenerate codons exactly matching the 
coevolving sites, were designed. For each pair of coevolving sites, PCR reactions were performed using two pairs of complementary primers, each pair corresponding to a coevolving site. After removal of the methylated template plasmid with DpnI enzyme, PCR products were transformed into E. coli XL1-Blue competent cells by chemical transformation [52]. The transformed cells harboring the CCSM libraries were plated on LB agar supplemented with antibiotics.

\section{Step 3: Screening of the improved mutants}

We used high throughput screening method to identify improved mutants from the CCSM library in a statistically significant way. In this study, mutant enzymes are assayed for residual activity relative to the wild-type strain after heat treatment and assayed for thermostability with respect to the half-inactivation temperature $\left(T_{50}^{30}\right)$. Clones demonstrating the highest thermostability and survival relative activity are rescreened, and the genes of rescreened variants are sequenced to identify the mutations. The identified mutant enzymes are purified, and the $T_{50}^{30}$ value and catalytic activity are further characterized to confirm the initial screening results.

The $\alpha$-amylase Amy7C [GenBank: JN980090], derived from Bacillus subtilis CN7, was used to demonstrate the utility of our CCSM method. Amy7C is $\mathrm{Ca}^{2+}$-independent and is relatively stable at a wide range of $\mathrm{pH}$ values. However, its thermostability is not sufficient for use in starch simultaneous saccharification and liquefaction processes. Bacillus subtilis CN7 was screened and deposited in our laboratory. The plate plasmid pSA7C, the host strains E. coli XL1-Blue and E. coli JM109, and nucleotide primers are listed in Table A1 (see Additional file 4: Table SA1). The primers were synthesized by Generay (Shanghai, China) and gene sequencing was performed by Shanghai DNA Biotechnologies (Shanghai, China). All the detailed materials and methods can be found in supporting materials (Additional file 1).

\section{Additional files}

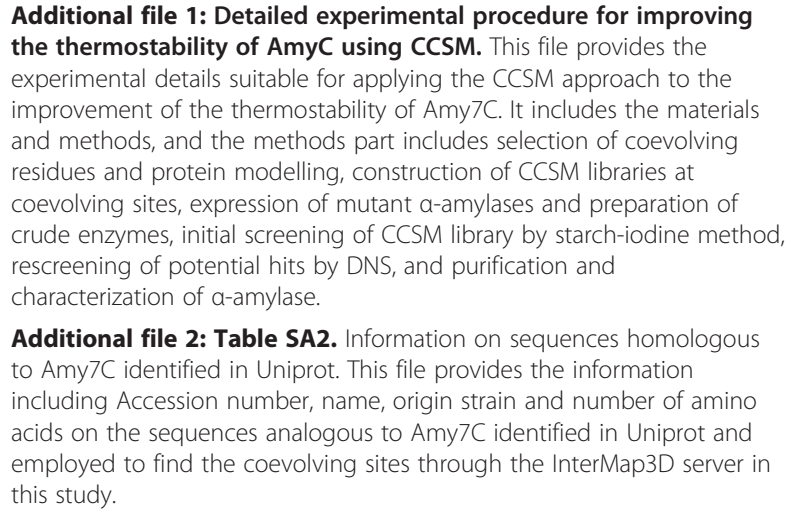

Additional file 2: Table SA2. Information on sequences homologous to Amy7C identified in Uniprot. This file provides the information including Accession number, name, origin strain and number of amino acids on the sequences analogous to Amy7C identified in Uniprot and employed to find the coevolving sites through the InterMap3D server in this study.

Additional file 3: Table SA3. Multiple Sequence Alignment in CLUSTAL format obtained by MAFFT method in this study. This file provides the Multiple Sequence Alignment in CLUSTAL format of the sequences homologous to Amy7C obtained by MAFFT method to find the coevolving sites through InterMap3D server in this study,

Additional file 4: Table SA1. Plasmids, strains, and primers used in this study. This file includes the plasmids, host strains, and nucleotide primers used in this study.

Additional file 5: Figure SA1. Close-up views of residues at coevolving sites in Amy7C and its variants. This file depicts the position and interaction of the residues at coevolving sites in Amy7C (A), H100l (B), D95HT147S (C), H100MD144R (D), T147P (E), N197C (F), and G89FD95R (G).

\section{Abbreviations}

CCSM: Combinatorial Coevolving-site Saturation Mutagenesis; RCW-MI: Row and Column Weighting of Mutual Information; HTS: High-Throughput Screening.

\section{Competing interests}

The authors declare that they have no competing interests.

\section{Authors' contributions}

CHW participated in the design of the study, carried out the molecular manipulations, interpreted the results and wrote the manuscript. RBH conceived of the study, performed data analysis and wrote the manuscript. QSD participated in data analysis and helped CHW to revise the manuscript. $\mathrm{BFH}$ helped CHW to implement the study and provided the guidance. All authors read and approved the final manuscript.

\section{Acknowledgements}

This work was supported by the Chinese National Basic Research Program ("973")[grant 2009CB724703] and National Science and Technology Support Program [grant 2007BAD75B05].

\section{Author details}

'Biotechnology and Pharmaceutical Engineering, Nanjing University of Technology, Xinmofan Road 5, Nanjing 210009, Jiangsu, China. ${ }^{2}$ State Key Laboratory of Enzyme Technology, National Engineering Research Center for Non-food Biorefinery, Guangxi Key Laboratory of Biorefinery, Guangxi Academy of Sciences, Nanning, Guangxi 530007, China. ${ }^{3}$ College of Life Science and Technology, Guangxi University, Nanning, Guangxi 530004, China.

Received: 21 March 2012 Accepted: 11 September 2012

Published: 11 October 2012

\section{References}

1. Yuan L, Kurek I, English J, Keenan R: Laboratory-directed protein evolution. Microbiol Mol Biol Rev 2005, 69(3):373-392.

2. Turner NJ: Directed evolution drives the next generation of biocatalysts. Nat Chem Biol 2009, 5(8):567-573.

3. Wong TS, Roccatano D, Schwaneberg U: Steering directed protein evolution: strategies to manage combinatorial complexity of mutant libraries. Environ Microbiol 2007, 9(11):2645-2659.

4. Reetz MT, Kahakeaw D, Lohmer R: Addressing the numbers problem in directed evolution. Chembiochem 2008, 9(11):1797-1804

5. Reetz MT, Soni P, Acevedo JP, Sanchis J: Creation of an amino acid network of structurally coupled residues in the directed evolution of a thermostable enzyme. Angew Chem Int Ed Engl 2009, 48(44):8268-8272.

6. Reetz MT, Wang LW, Bocola M: Directed evolution of enantioselective enzymes: iterative cycles of CASTing for probing protein-sequence space. Angew Chem Int Ed Engl 2006, 45(8):1236-1241.

7. Reetz MT, Carballeira JD, Vogel A: Iterative saturation mutagenesis on the basis of $B$ factors as a strategy for increasing protein thermostability. Angew Chem 2006, 118(46):7909-7915.

8. Reetz MT, Carballeira JD: Iterative saturation mutagenesis (ISM) for rapid directed evolution of functional enzymes. Nat Protoc 2007, 2(4):891-903.

9. Reetz MT, Bocola M, Carballeira JD, Zha D, Vogel A: Expanding the range of substrate acceptance of enzymes: combinatorial active-site saturation test. Angew Chem Int Ed Engl 2005, 44(27):4192-4196. 
10. Wiederstein M, Sippl MJ: Protein sequence randomization: efficient estimation of protein stability using knowledge-based potentials. J Mol Biol 2005, 345(5):1199-1212.

11. Hayes RJ, Bentzien J, Ary ML, Hwang MY, Jacinto JM, Vielmetter J, Kundu A, Dahiyat Bl: Combining computational and experimental screening for rapid optimization of protein properties. Proc Natl Acad Sci U S A 2002, 99(25):15926-15931.

12. Voigt CA, Mayo SL, Arnold FH, Wang ZG: Computational method to reduce the search space for directed protein evolution. Proc Natl Acad Sci U S A 2001, 98(7):3778-3783.

13. Armon A, Graur D, Ben-Tal N: ConSurf: an algorithmic tool for the identification of functional regions in proteins by surface mapping of phylogenetic information. J Mol Biol 2001, 307(1):447-463.

14. Pollock DD, Taylor WR, Goldman N: Coevolving protein residues: maximum likelihood identification and relationship to structure. J Mol Biol 1999, 287(1):187-198

15. Yeang $\mathrm{CH}$, Haussler D: Detecting coevolution in and among protein domains. PLoS Comput Biol 2007, 3(11):e211.

16. Ferrer-Costa C, Orozco M, de la Cruz X: Characterization of compensated mutations in terms of structural and physico-chemical properties. $J \mathrm{Mol}$ Biol 2007, 365(1):249-256.

17. Lockless SW, Ranganathan R: Evolutionarily conserved pathways of energetic connectivity in protein families. Science 1999, 286(5438):295-299.

18. Hatley ME, Lockless SW, Gibson SK, Gilman AG, Ranganathan R: Allosteric determinants in guanine nucleotide-binding proteins. Proc Natl Acad SCi U S A 2003, 100(24):14445-14450.

19. Chakrabarti S, Panchenko AR: Coevolution in defining the functional specificity. Proteins 2009, 75(1):231-240.

20. Gobel U, Sander C, Schneider R, Valencia A: Correlated mutations and residue contacts in proteins. Proteins 1994, 18(4):309-317.

21. Lee BC, Park K, Kim D: Analysis of the residue-residue coevolution network and the functionally important residues in proteins. Proteins 2008, 72(3):863-872

22. Gouveia-Oliveira R, Pedersen AG: Finding coevolving amino acid residues using row and column weighting of mutual information and multidimensional amino acid representation. Algorithms Mol Biol 2007, 2:12.

23. Dutheil J, Galtier N: Detecting groups of coevolving positions in a molecule: a clustering approach. BMC Evol Biol 2007, 7:242

24. Fares MA, McNally D: CAPS: coevolution analysis using protein sequences. Bioinformatics 2006, 22(22):2821-2822

25. Gao H, Dou Y, Yang J, Wang J: New methods to measure residues coevolution in proteins. BMC Bioinformatics 2011, 12:206.

26. Gloor GB, Martin LC, Wahl LM, Dunn SD: Mutual information in protein multiple sequence alignments reveals two classes of coevolving positions. Biochemistry 2005, 44(19):7156-7165

27. Pollock DD: Genomic biodiversity, phylogenetics and coevolution in proteins. Appl Bioinformatics 2002, 1(2):81-92.

28. Codoñer FM, Fares MA: Why should we care about molecular coevolution? Evol Bioinform 2008, 4:29-38.

29. Prakash O, Jaiswal N: Alpha-Amylase: an ideal representative of thermostable enzymes. App/ Biochem Biotechnol 2010, 160(8):2401-2414.

30. Hocker B, Jurgens C, Wilmanns M, Sterner R: Stability, catalytic versatility and evolution of the (beta alpha)(8)-barrel fold. Curr Opin Biotechnol 2001, 12(4):376-381.

31. Declerck N, Machius M, Joyet P, Wiegand G, Huber R, Gaillardin C Engineering the thermostability of Bacillus licheniformis a-amylase. Biologia, Bratislava 2002, 57(Suppl. 11):203-211.

32. Little DY, Chen L: Identification of coevolving residues and coevolution potentials emphasizing structure, bond formation and catalytic coordination in protein evolution. PLOS One 2009, 4(3):e4762.

33. Fuwa $\mathrm{H}$ : A new method for microdetermination of amylase activity by the use of amylose as the substrate. J Biochem 1954, 41(5):583-603.

34. Miller $G L$ : Use of dinitrosalicylic acid reagent for determination of reducing sugar. Anal Chem 1959, 31(3):426-428.

35. Sterner $\mathrm{R}$, Hocker B: Catalytic versatility, stability, and evolution of the (betaalpha)8-barrel enzyme fold. Chem Rev 2005, 105(11):4038-4055.

36. Gromiha MM, Pujadas G, Magyar C, Selvaraj S, Simon I: Locating the stabilizing residues in (alpha/beta) 8 barrel proteins based on hydrophobicity, long-range interactions, and sequence conservation. Proteins 2004, 55(2):316-329.
37. Nagatani RA, Gonzalez A, Shoichet BK, Brinen LS, Babbitt PC: Stability for function trade-offs in the enolase superfamily "catalytic module". Biochemistry 2007, 46(23):6688-6695

38. Declerck N, Machius M, Joyet P, Wiegand G, Huber R, Gaillardin C: Hyperthermostabilization of Bacillus licheniformis alpha-amylase and modulation of its stability over a 50 degrees $\mathrm{C}$ temperature range. Protein Eng 2003, 16(4):287-293.

39. Declerck N, Joyet P, Gaillardin C, Masson JM: Use of amber suppressors to investigate the thermostability of Bacillus licheniformis alpha-amylase. Amino acid replacements at 6 histidine residues reveal a critical position at His-133. J Biol Chem 1990, 265(26):15481-15488.

40. Joyet P, Declerck N, Gaillardin C: Hyperthermostable variants of a highly thermostable alpha-amylase. Biotechnology (N Y) 1992, 10(12):1579-1583.

41. Declerck N, Joyet P, Trosset JY, Garnier J, Gaillardin C: Hyperthermostable mutants of Bacillus licheniformis alpha-amylase: multiple amino acid replacements and molecular modelling. Protein Eng 1995, 8(10):1029-1037.

42. Declerck N, Machius M, Chambert R, Wiegand G, Huber R, Gaillardin C: Hyperthermostable mutants of Bacillus licheniformis alpha-amylase: thermodynamic studies and structural interpretation. Protein Eng 1997, 10(5):541-549.

43. Declerck N, Machius M, Wiegand G, Huber R, Gaillardin C: Probing structural determinants specifying high thermostability in Bacillus licheniformis alpha-amylase. J Mol Biol 2000, 301(4):1041-1057.

44. Machius M, Declerck N, Huber R, Wiegand G: Kinetic stabilization of Bacillus licheniformis alpha-amylase through introduction of hydrophobic residues at the surface. J Biol Chem 2003, 278(13):11546-11553.

45. Whittle E, Shanklin J: Engineering delta 9-16:0-acyl carrier protein (ACP) desaturase specificity based on combinatorial saturation mutagenesis and logical redesign of the castor delta 9-18:0-ACP desaturase. J Biol Chem 2001, 276(24):21500-21505.

46. Apweiler R, Bairoch A, Wu CH, Barker WC, Boeckmann B, Ferro S, Gasteiger E, Huang H, Lopez R, Magrane M, et al: UniProt: the Universal Protein knowledgebase. Nucleic Acids Res 2004, 32:D115-D119.

47. Katoh $\mathrm{K}$, Kuma $\mathrm{K}$, Toh $\mathrm{H}$, Miyata T: MAFFT version 5 : improvement in accuracy of multiple sequence alignment. Nucleic Acids Res 2005, 33(2):511-518.

48. Gouveia-Oliveira R, Sackett PW, Pedersen AG: MaxAlign: maximizing usable data in an alignment. BMC Bioinformatics 2007, 8:312.

49. Gouveia-Oliveira R, Roque FS, Wernersson R, Sicheritz-Ponten T, Sackett PW, Molgaard A, Pedersen AG: InterMap3D: predicting and visualizing coevolving protein residues. Bioinformatics 2009, 25(15):1963-1965.

50. InterMap3D server. http://www.cbs.dtu.dk/services/InterMap3D/.

51. Hogrefe $\mathrm{HH}$, Cline J, Youngblood GL, Allen RM: Creating randomized amino acid libraries with the QuikChange multi site-directed mutagenesis kit. Biotechniques 2002, 33(5):1158-1160. 1162, 1164-1155.

52. Sambrook J, Russell D: Molecular Cloning: A Laboratory Manual third edn. New York: Cold Spring Harbor Laboratory Press; 2001.

doi:10.1186/1471-2105-13-263

Cite this article as: Wang et al:: Improving the thermostability of alphaamylase by combinatorial coevolving-site saturation mutagenesis. BMC Bioinformatics 2012 13:263.

\section{Submit your next manuscript to BioMed Central and take full advantage of:}

- Convenient online submission

- Thorough peer review

- No space constraints or color figure charges

- Immediate publication on acceptance

- Inclusion in PubMed, CAS, Scopus and Google Scholar

- Research which is freely available for redistribution 